Meta

Journal des traducteurs

Translators' Journal

\title{
Traduire le proverbe : à la recherche de concordances parémiologiques en bété pour la constitution d'un corpus trilingue allemand/français/bété
}

\author{
Jean Philippe Zouogbo
}

Volume 53, numéro 2, juin 2008

La traduction des séquences figées

The Translation of Frozen Sequences

URI : https://id.erudit.org/iderudit/018521ar

DOI : https://doi.org/10.7202/018521ar

Aller au sommaire du numéro

Éditeur(s)

Les Presses de l'Université de Montréal

ISSN

0026-0452 (imprimé)

1492-1421 (numérique)

Découvrir la revue

Citer cet article

Zouogbo, J. P. (2008). Traduire le proverbe : à la recherche de concordances parémiologiques en bété pour la constitution d'un corpus trilingue allemand/français/bété. Meta, 53(2), 310-323. https://doi.org/10.7202/018521ar

\section{Résumé de l'article}

La problématique de la traduction du proverbe d'une langue de départ à une langue cible constitue un aspect focal des travaux que nous avons menés antérieurement. L'une des étapes de cette recherche ayant été la constitution d'un corpus parallèle de proverbes en trois langues, l'allemand, le français et le bété, une langue ivoirienne (Zouogbo 2005). L'existence de lexiques dans les langues à rayonnement international et la facilité qu'il y a, à partir de sources lexicographiques pour trouver aux proverbes d'une langue A des correspondants dans une langue $\mathrm{B}$, a grandement contribué à passer sous silence, sinon à occulter ces longs et complexes mécanismes cognitifs qui permettent de transposer la pensée imageante d'une langue à une autre. En effet, la première étape de la constitution de nos corpora consistait à prendre pour point de repère un corpus expérimental de proverbes allemands en vue de trouver des concordances en français. Elle s'est avérée relativement aisée parce que nous nous sommes appuyé sur des sources existantes. Nous avons, au contraire, réalisé dans le second volet de notre travail de répertorisation et de transcription des proverbes bété en allemand et en français qu'il s'agissait là d'une tâche hautement délicate et complexe. Car selon quelles modalités fallait-il non seulement transcrire les unités recueillies à l'oral mais aussi, comment parvenir à trouver et à conférer à ces formes orales un contenu et une morphologie identiques aux proverbes des autres langues ? Car un proverbe, en dépit de sa structure, est une unité linguistique encore loin d'être un simple énoncé phrastique?

Nous avons ainsi rendu compte des différents mécanismes pris en considération pour la constitution de notre concordancier en bété et en particulier les techniques mises en oeuvre pour trouver aux proverbes allemands et français des correspondants en bété. Une méthodologie pouvant servir aussi pour d'autres langues en contraste avec des langues étalons. Ont également été abordées les caractéristiques morphologiques et sémantiques du proverbe et, de façon générale, la distinction entre les proverbes prototypiques et les parémies.
Ce document est protégé par la loi sur le droit d'auteur. L’utilisation des services d’Érudit (y compris la reproduction) est assujettie à sa politique d'utilisation que vous pouvez consulter en ligne.

https://apropos.erudit.org/fr/usagers/politique-dutilisation/ 


\title{
Traduire le proverbe: à la recherche de concordances parémiologiques en bété pour la constitution d'un corpus trilingue allemand/français/bété
}

\author{
JEAN PHILIPPE ZOUOGBO \\ Université Paris-Diderot/Paris 7, Paris, France \\ zouogbo@hotmail.com
}

\section{RÉSUMÉ}

La problématique de la traduction du proverbe d'une langue de départ à une langue cible constitue un aspect focal des travaux que nous avons menés antérieurement. L'une des étapes de cette recherche ayant été la constitution d'un corpus parallèle de proverbes en trois langues, l'allemand, le français et le bété, une langue ivoirienne (Zouogbo 2005). L'existence de lexiques dans les langues à rayonnement international et la facilité qu'il y a, à partir de sources lexicographiques pour trouver aux proverbes d'une langue $A$ des correspondants dans une langue $B$, a grandement contribué à passer sous silence, sinon à occulter ces longs et complexes mécanismes cognitifs qui permettent de transposer la pensée imageante d'une langue à une autre. En effet, la première étape de la constitution de nos corpora consistait à prendre pour point de repère un corpus expérimental de proverbes allemands en vue de trouver des concordances en français. Elle s'est avérée relativement aisée parce que nous nous sommes appuyé sur des sources existantes. Nous avons, au contraire, réalisé dans le second volet de notre travail de répertorisation et de transcription des proverbes bété en allemand et en français qu'il s'agissait là d'une tâche hautement délicate et complexe. Car selon quelles modalités fallait-il non seulement transcrire les unités recueillies à l'oral mais aussi, comment parvenir à trouver et à conférer à ces formes orales un contenu et une morphologie identiques aux proverbes des autres langues? Car un proverbe, en dépit de sa structure, est une unité linguistique encore loin d'être un simple énoncé phrastique?

Nous avons ainsi rendu compte des différents mécanismes pris en considération pour la constitution de notre concordancier en bété et en particulier les techniques mises en œuvre pour trouver aux proverbes allemands et français des correspondants en bété. Une méthodologie pouvant servir aussi pour d'autres langues en contraste avec des langues étalons. Ont également été abordées les caractéristiques morphologiques et sémantiques du proverbe et, de façon générale, la distinction entre les proverbes prototypiques et les parémies.

\section{ABSTRACT}

Focusing our research on the translation of proverbs from one language to another, our goal has been to build up a parallel corpus of proverbs in three languages: German, French and Bété, from the Ivory Coast. The translation of orally quoted Bété proverbs was particularly difficult because there is no paremiological written source, which is not the case for the other languages.

The fact that it is easy to find proverb glossaries in international influent languages and, from these glossaries, to find paremiological correspondents has been a major factor in concealing those long and complicated cognitive mecanisms which are instrumental in conveying the figurative thought from language A to language B. A proverb, in spite of its morphological and syntactic structure, is a language unit which stands apart 
from an ordinary sentence. It is still a phrase based on propositional content and on morphological and syntactical structures. Thus, translating a proverb also entails the transfer of not only the idea or meaning of the fixed phrase, but also, and as far as possible, the structure.

In this article, we try to devise a method of translation for paremiological units to assist linguists who collect proverbs from languages considered as minor and compare them with those of the international influent languages. We have also analyzed the metaphorical proverb itself with special attention to its semantic and morphological characteristics.

\section{MOTS-CLÉS/KEYWORDS}

allemand, bété, concordances parémiologiques, proverbe

\section{Introduction}

Le mérite revient aux recherches portant sur les phénomènes langagiers et, en particulier, aux études des phraséologues, d'avoir consacré le proverbe, non seulement comme un fait de langue à part entière, mais aussi et surtout comme un phraséologisme. En tant qu'il est élément de cette vaste catégorie, le proverbe est solidaire des unités phraséologiques du point de vue des difficultés rencontrées par les chercheurs lorsqu'il s'agit de les étudier. Et l'un des principaux obstacles liés au traitement de ces unités demeure la traduction. Non pas qu'il s'agisse d'unités intraduisibles, mais parce que, eu égard à leur statut morphologique et sémantique, leur traitement traductologique procède nécessairement de la prise en compte de la structure et des caractéristiques dont relèvent les phraséologismes et, pour ainsi dire, le proverbe.

La problématique de la traduction du proverbe d'une langue de départ à une langue cible constitue un aspect focal des travaux que nous avons menés antérieurement. L'une des étapes de cette recherche ayant été la constitution d'un corpus parallèle de proverbes en trois langues, l'allemand, le français et le bété, une langue ivoirienne (Zouogbo 2005). L'existence de lexiques dans les langues à rayonnement international et la facilité qu'il y a à partir de sources lexicographiques pour trouver aux proverbes d'une langue A des correspondants dans une langue B a grandement contribué à passer sous silence, sinon à occulter ces longs et complexes mécanismes cognitifs qui permettent de transposer la pensée imageante d'une langue à une autre. En effet, si la première étape de la constitution de nos corpora qui consistait à prendre pour point de repère un corpus expérimental de proverbes allemands en vue de trouver des concordances en français s'est avérée relativement aisée parce que nous nous sommes appuyé sur des sources existantes, nous avons réalisé dans le second volet de notre travail de répertorisation et de transcription des proverbes bété en allemand et en français qu'il s'agissait là d'une tâche hautement délicate et complexe. Car selon quelles modalités fallait-il non seulement transcrire les unités recueillies à l'oral mais aussi, comment parvenir à trouver et à conférer à ces formes orales un contenu et une morphologie identiques aux proverbes des autres langues? Car est-il besoin de souligner qu'un proverbe, en dépit de sa structure, est loin d'être un simple énoncé phrastique?

Dans les développements qui suivent, nous rendrons compte des différents mécanismes pris en considération pour la constitution de notre concordancier en bété et en particulier les techniques mises en œuvre pour trouver aux proverbes allemands 
et français des correspondants en bété. Seront également abordées, bien entendu, les caractéristiques morphologiques et sémantiques du proverbe et, de façon générale, la distinction entre les proverbes prototypiques et les parémies. La mise en contraste de quelques proverbes issus des trois langues de travail de notre corpus permettra en outre de dégager la nature des concordances parémiologiques constatées.

\section{Le proverbe, cet autre figement}

\subsection{Le proverbe au crible des critères phraséologiques}

Les critères définitoires des phraséologismes sont la polylexicalité, le figement et la figuration. Les deux premiers sont obligatoires pour qu'une collocation soit qualifiée de phrasème. Le troisième, la figuration, n'est pas énoncé pour l'ensemble des séquences figées. Par polylexicalité, on entend le regroupement d'au moins deux lexèmes. On dit des phrasèmes qu'ils sont figés parce que c'est avec leur morphologie actuelle qu'ils sont intégrés dans le lexique, et que les locuteurs les découvrent, exactement, comme ils découvriraient un lexème. La figuration est, chez Gréciano (1984), la répercussion sémantique de la polylexicalité et du figement. On dira qu'un phrasème est figuré lorsque le sens du syntagme figé n'est pas fonction de la somme des monèmes qui le composent. Ainsi, avec Es kräht kein Hahn danach; Nach Kanossa gehen; Le coup de pied de l'âne; Rat d'hôtel, on peut parler de figuration. Et c'est ce principe qui s'applique aux unités que l'on nomme proverbe. C'est la figuration qui permet de passer de la lecture stable et innocente d'un syntagme comme Tout ce qui brille n'est pas de l'or au contenu conceptuel des APPARENCES TROMPEUSES et qui fait que ce syntagme devient, par extension, un proverbe. En tant que tel, si on élude la dénotation seconde du syntagme (à savoir le contenu conceptuel véhiculé), Tout ce qui brille n'est pas de l'or n'est rien de plus qu'un simple énoncé minéralogique. En effet, les lexèmes constitutifs du phrasème sont des signes qui possèdent une valeur sémantique autonome à travers lesquels on les reconnaît. Lorsqu'ils sont mis en combinatoire, ils perdent leurs valeurs respectives pour se mettre au service de la valeur sémantique de la nouvelle entité dont ils deviennent les constituants. Gréciano parle alors d'une démotivation obligatoire (qui est) la perte du sens littéral: Gibt man dem Teufel den kleinen Finger [...] vs Mieux vaut prévenir que guérir.

Dans leur ensemble, les phrasèmes peuvent être décrits selon trois niveaux: ils sont référentiels, structurels et/ou communicatifs (Burger 2003).

Les proverbes sont classés au titre des phrasèmes référentiels parce qu'ils ont un rapport avec des objets, des actions et des faits, que ceux-ci soient fictifs ou réels. Cependant, pour quelque phraséologiques qu'ils soient, les proverbes ne possèdent pas moins des traits propres qui permettent de les démarquer de la classe des phrasèmes en général. Dénotons-en quelques-uns: il existe, en effet, des unités phraséologiques qui partagent avec le proverbe les mêmes propriétés lexicales et syntaxiques. Il s'agit, en réalité, des phrasèmes dits syntagmes figés ou phrases figées, locutions propositionnelles / feste Phrasen comme:

Mit ihm kann man ein Pferd stehlen.

Wo der hinhaut, da wächst kein Gras mehr.

C'est la goutte d'eau qui fait déborder le vase.

Il est connu comme le loup blanc. 
Nous partons de l'hypothèse selon laquelle les proverbes sont des phrasèmes propositionnels. Il s'agit de phrases dotées d'une autonomie qui leur permet de conserver leur forme lexicale lorsqu'elles sont insérées dans la chaîne. Leur compréhension et leur rôle dans le texte, cependant, sont fonction du contexte d'emploi. Syntagmes figés et proverbes sont également dits phrasèmes référentiels: ils sont dénominatifs en ce sens que leur fonction dans le discours permet de renvoyer à des concepts et objets du monde, réels ou virtuels, d'exprimer des faits psychologiques et mentaux. Ils sont généralement idiomatiques car leur sens n'est pas sommable à partir des constituants. La parenté entre proverbes et autres phrasèmes s'arrête au niveau de la dénotation des objets et des concepts, car les proverbes, en outre, sont des stéréotypes de certaines situations, de certaines réalités et sont conçus comme modèles. Qui plus est, contrairement aux syntagmes figés et aux autres formes de phrasèmes, les proverbes ont une valeur morale ou éthique et sont des formules topiques. Les autres phrasèmes sont certes généralement idiosyncrasiques mais ne sont pas des formules idéelles complètes et forcement compréhensibles a priori. En plus, elles ne vont pas au-delà de la structure phrastique. Les formules topiques, quant à elles, peuvent être des (micro)textes entiers qui contiennent implicitement des thèmes dont ils traitent et dans lesquels on retrouve des faits tenus pour vrais, car relevant de l'expérience pratique et populaire et qui sont liés à un groupe culturel donné.

La généricité permet de dissocier les proverbes de syntagmes comme c'est la goutte d'eau qui fait déborder le vase qui sont des phrases situationnelles parce que, bien que servant à caractériser une situation (généricité), elles sont, contrairement au proverbe, épisodiques (Anscombre 2003: 11). En outre, l'énoncé générique possède un auteur, l'énonciateur premier, même si celui-ci n'est pas ou plus connu. Celui qui reprend le proverbe n'en étant que l'énonciateur second, c'est-à-dire celui qui porte un jugement à travers la parole d'un autre (l'énonciateur premier). Mais la différence va plus loin. Les phrasèmes, qui ne disposent pas des deux degrés d'énonciation (première et seconde), se limitent au statut de phrases génériques premier locales "parce qu'ils servent à leur locuteur à émettre un jugement propre» (référence) alors que les proverbes qui ont un énonciateur spécifique premier et un énonciateur second sont des phrases génériques premier a priori.

Le statut de figement lexical et syntaxique des proverbes tient au fait que le locuteur utilise des phrases toutes faites pour signifier un fait qu'il aurait bien pu décrire et exprimer à travers ses propres mots. Ils sont donc connus et appris sous la forme lexicale qu'on leur connaît et réutilisés sous cette même forme. Qui plus est, le proverbe marque une distanciation nette du locuteur avec le contexte d'emploi actuel. En effet, le locuteur en situation d'encodage l'utilise en toute connaissance de cause pour dénommer un fait qui lui tient à cœur. En optant pour un proverbe plutôt que de compter sur sa compétence linguistique personnelle, il se réfugie derrière une formule connue et de portée générale. Il exprime ouvertement un fait sans toutefois en être l'auteur. Aussi l'usage parfait d'un proverbe sera-t-il jugé à travers l'adéquation entre le syntagme utilisé, l'état psychologique du locuteur et la situation d'encodage. Ce sont ces facteurs qui permettront à l'allocutaire de saisir le message véhiculé et de permettre la réalisation pleine de l'acte de communication. La condition principale chez les interlocuteurs est qu'ils soient en mesure de décoder le contenu plus ou moins idiomatique du proverbe. Ceci présuppose une bonne saisie de l'arrière-plan culturel. Ce qui n'est pas une condition nécessaire pour la compréhension des autres phrasèmes. 
Si les critères de la polylexicalité, du figement et de la figuration (la figuration fait partie du figement?) confèrent au proverbe son statut de phraséologisme, il convient de retenir que toutes les unités considérées par la recherche de ces dernières années comme étant un proverbe ne le sont pas en réalité. Car ne sont considérées comme proverbes que les séquences ayant un aspect réellement métaphorique. C’est en réalité en regard de ces entités-là que le traducteur se trouve interpellé. Car leur signification est bien souvent contextuelle et transphrastique, c'est-à-dire, tout comme les idiomes, en rupture totale avec la simple association de sens des constituants qui les composent. En tant que tel, il n'est pas inutile de distinguer entre parémies (toutes les formules sapientales) et proverbe (les parémies métaphoriques, celles-là mêmes qui sont considérées comme les prototypes du proverbe).

\subsection{Le proverbe prototypique: le meilleur exemplaire de sa catégorie}

Appuyons-nous sur Rodegem (1984) et Buridant (1984) pour mieux saisir le démêlage notionnel en vigueur en parémiologie.

Rodegem (1984) a contribué à établir une échelle de valeurs entre toutes les formules sapientiales. Il substitue désormais à «ce qu'on appelle généralement proverbes», le terme de parémie "pour éviter des ambiguïtés». Il définit la parémie comme «une sentence lapidaire normative » (référence; page) dont les critères constitutifs sont la morphologie rythmique, la structure analogique et le statut normatif. Il recense dix formules gnomiques, qu'il nomme parémies, et qu'il classe en trois groupes: le premier, dont les éléments constitutifs expriment une morale au sens général, une norme générale, est constitué par:

1. le proverbe: Le chat sauvage ne sait pas où il a pris une poule.

2. la locution proverbiale: Crier famine sur un tas de blé.

3. la maxime: Qui ne risque rien n'a rien.

4. l'aphorisme: Tel père, tel fils.

Le deuxième groupe est composé de parémies qui sont censées, selon Rodegem, exprimer une norme restreinte:

5. le dicton: Noël au balcon, Pâques au tison.

6. l'adage: En fait de biens, possession vaut titre.

7. le slogan: Santé de fer.

8. la devise: Honni soit qui mal y pense.

Pour le troisième groupe, il s'agit, dans la pensée de Rodegem, de genres marginaux:

9. l'apophtegme: La critique est aisée mais l'art est difficile.

10. le wéllerisme: "La vertu au milieu» comme disait le diable en se mettant entre deux prostituées.

Buridant apporte une approche innovante dans la délimitation terminologique des parémies en définissant des traits d'identification et des traits de spécification. Les traits d'identification du proverbe sont repérables à la structure morphologique de la formule (qui peut être soit un énoncé autonome, soit un microtexte) et au procédé d'intégration dans les discours, où les marqueurs métalinguistiques (comme on dit, La sagesse affirme que...) jouent un rôle important. Les traits de spécification entrent en jeu à partir du moment où on évoque les variantes des proverbes. La métaphore 
constitue un des principaux traits de spécification des parémies. Buridant approuve également l'hypéronyme parémie et soutient que seule pareille dénomination peut éviter les «flous terminologiques». On retrouvera ainsi chez Buridant (1976) trois critères parémiologiques: le rythme, la norme et la figuration. Les parémies ne se conformant pas à ces critères ne sont pas des proverbes. Cette différenciation moins complexe a pour avantage de permettre d'établir une distinction nette entre le proverbe et les notions voisines. Nous en retiendrons pour notre part, cependant, que le critère de la figuration. En effet, nous considérons toutes ces dénominations comme les éléments d'une même catégorie. Le seul critère que nous retenons est celui de la métaphoricité. Les syntagmes métaphoriques étant les prototypes de la catégorie des parémies.

\section{Traduire le proverbe ou les enjeux de la constitution d'un corpus parallèle}

Les analyses qui précèdent font état de la non-compositionnalité du proverbe au même titre que les phrasèmes. En tant que tel, traduire le proverbe ne se réduit plus au simple mécanisme de la transposition littérale dans une langue $B$ d'une séquence énoncée dans une langue A. Pour traduire les proverbes du bété, répertoriés à l'oral, et pour leur conférer une forme proche des proverbes allemands et français, nous avons dû tenir compte de plusieurs paramètres. Au nombre de ceux-ci apparaissent la prise en compte du contenu propositionnel des proverbes et leur morphologie. Car, d'une part, le proverbe n'est considéré comme tel que quand il convoie de façon idiomatique un contenu conceptuel au moyen d'images tirées du fonds commun et de l'arrière-plan culturel. Et évoquer en outre l'universalité des proverbes (facteur qui justifie, dans plusieurs études, la recherche des affinités et des dissemblances dans des langues différentes sur la base de leur trésor parémiologique), ce n'est pas non plus de se limiter au seul exposé du contenu sémantique des proverbes. La morphologie joue un rôle tout aussi important dans la lecture du proverbe. C'est, pour ainsi dire, par le contenu et également par la forme que le proverbe se démarque des autres formulations phrastiques. Ainsi, en réécrivant un proverbe d'une langue non encore élaborée, il importe de traduire non seulement le contenu propositionnel, mais il faut aussi veiller à conférer à cette nouvelle formule une morphologie qui garde les aspects stylistiques conventionnels. Nos travaux ont démontré que les proverbes du bété (langue non véritablement élaborée) procèdent des mêmes morphologies que ceux de l'allemand ou du français (Zouogbo 2005). Dans ce qui suit, nous apporterons un éclairage succinct sur les notions de concepts et d'images qui participent du contenu propositionnel des proverbes et nous exposerons les principaux moules morphologiques des parémies conventionnellement admis.

\subsection{Les concepts et les images parémiologiques}

Le proverbe n'est lisible qu'à travers le concept qu'il exprime. Le concept parémiologique est une idée abstraite et générale qui est déduite du proverbe. C’en est même la conséquence immédiate sur le plan sémantique car l'expression idiomatique, et, dans notre cas, le proverbe, est un signe au sens saussurien de l'union entre image acoustique et sens. Et celui-ci est souvent figuré. Le sens du proverbe, en effet, ne procède 
donc pas de la compositionnalité de ses formatifs. C'est le cas ici dans les exemples qui suivent, où est dénotée la VANITÉ, ou plus précisément dans le second, l'OUTRECUIDANCE.

Leere Fässer klingen hohl (Beyer, 147)

Après le crépuscule, les vers luisants disent: "Nous avons donné la lumière au monde.» (Maloux, 39)

Le proverbe est, en effet, une phrase générique dont le sens lexical des éléments colloqués est suspendu au profit de la valeur sémantique de l'ensemble. C'est le concept qui confère au proverbe son identité sémantique. Le concept est donc vu comme le noyau sémantique de l'expression parémiologique.

Les images parémiologiques, quant à elles, résultent de la conjonction de processus métaphoriques et de figement de certains formatifs dans le code culturel et langagier propre à chaque langue. Le traitement de l'image en parémiologie implique, pour ainsi dire, la nécessaire prise en compte de plusieurs facteurs liés à la nature des constituants nominaux porteurs de ces images, à leurs domaines sources, au processus de transfert de sens qui fait d'une entité quelconque un signe porteur de signification particulière et surtout à la fonction symbolique assignée à chacun des constituants porteurs d'image.

Les proverbes dont le signifié réel n'est déductible que par la motivation des constituants sont ceux que nous appellerons proverbes imagés. Il s'agit, ainsi que nous l'avons souligné, d'entités considérées comme prototypiques de la catégorie des parémies parce qu'elles obéissent suffisamment aux critères de figuration et de noncompositionnalité lexicales. Elles se dédoublent en outre d'une remotivation iconique, ce qui fait du syntagme parémiologique un énoncé différent de toute autre formulation phrastique. En effet, en considérant les exemples suivants:

(1) Der ${ }^{\star}$ Krug geht so lange $z u{ }^{\star}$ Wasser, bis er bricht (95) Tant va la cruche à *'eau qu'à la fin elle se brise. MPS53

(2) ${ }^{\star}$ Alte Bäume biegt man nicht mehr (Beyer, 34) Le ${ }^{*}$ cheval indompté devient intraitable. (Maloux 147)

on s'aperçoit que lesdits énoncés sont des constructions phrastiques ordinaires qui ne transgressent aucune règle de syntaxe. Seulement, l'énoncé parémiologique dépasse ce premier stade, car l'extension d'un pareil énoncé est plus englobante. Les séquences figées présentent ici des constructions sémantiques en rupture totale avec la norme retenue par la logique de l'entendement humain (une cruche qui se déplacerait d'elle-même par exemple!). Dans les proverbes, ce sont les signes linguistiques tels que Bäume / arbre; Krug / cruche... que l'on nomme en effet images. Il ne s'agit ici, bien entendu, pas d'image au sens audiovisuel mais vue sous un angle cognitif. C'est justement cette anthropomorphisation d'éléments appartenant à notre environnement, par processus métaphorique ou métonymique conventionnels dans les proverbes, qui confère à ces entités un statut d'image. Car les images en question dans les proverbes ne sont qu'accessoirement signifiantes pour elles-mêmes, elles sont des allusions à un extralinguistique qu'elles symbolisent. C'est donc en raison de leur symbolisme, c'est-à-dire la vision, l'opinion que l'Homme se fait d'eux et, à travers eux, de lui-même, qu'on nomme certains formatifs des images. Ces signes, tirés de domaines concrets, transcendent, en effet, leur statut linguistique premier, celui de signifiant pour un objet donné de l'univers, pour référer, par un processus de trans- 
fert de sens, à une situation autre que celle prédiquée par l'énoncé. Il s'agit généralement alors de moyens linguistiques pour mettre en évidence un certain nombre de représentations et stigmatiser une réalité extralinguistique, en l'occurrence un individu et ses traits distinctifs qui le caractérisent. Les images parémiologiques sont, pour ainsi dire, le fruit de l'expérience humaine qu'elles essaient, à leur tour, de rendre manifeste et de pérenniser. Ainsi, c'est l'Homme qui est lui-même à la base de la naissance des images qui à leur tour réfèrent à lui.

Une image dans un proverbe est considérée comme telle parce qu'elle ramène au présent et réactualise une situation ou un état qui a été envisagé, vécu ou éprouvé dans le passé. Un signifiant concret devient une image parce qu'il possède dorénavant une portée symbolique. L'image ne reflète alors donc plus, en réalité, le formatif porteur de l'image mais bien l'idée que se fait l'Homme de cette entité de l'univers, qu'il s'agisse de chien, pomme, fleuve ou oil... et, en définitive, l'idée qu'il se fait de lui-même. C'est ce transfert d'une situation concrète, d'un domaine source à un autre, à travers un usage fictif, qui fait la perspicacité des images parémiologiques.

\subsection{La morphosyntaxe du proverbe}

D'un point de vue morphologique, les proverbes reviennent régulièrement sous l'une ou l'autre forme. Seiler (1922: 186-194) avait établi, pour les proverbes allemands, une typologie selon quatorze moules. Ces mêmes constructions se retrouvent en français et certainement dans toutes les langues naturelles (Zouogbo 2005):

1. L'utilisation de l'hypotaxe:

- Wer etwas tut, muss etwas erleiden / Qui a fait ceci, doit subir cela.

Wer sich selbst wegwirft, den heben auch die anderen nicht auf (40)

Qui se fait agneau, le loup le mange. (Maloux 141)

- Wer etwas tut oder getan hat, der muss auch etwas tun / celui qui fait ou a fait ceci, devra faire cela: c'est la relation de cause à effet dans le sens où l'individu ayant posé un acte doit en assumer les conséquences:

Wer den Karren in den Dreck geschoben hat, muss ihn wieder herausziehen (261)

Qui invite les cigognes doit avoir des grenouilles. MPS232

2. L'usage fréquent de corrélatifs je-je (desto) / plus...plus:

Je giftiger der Pilz, je schöner die Kappe (219)

Plus on remue la boue, plus elle pue. (Maloux 88)

3. L'usage formel de la négation selon quatre procédés dont: kein-ohne / pas

de... - sans

Keine Rose ohne Dornen (109)

Keine Freud ohne Leid (173)

Il n'y a pas de roses sans épines

Il n'y a pas de fumée sans feu

la non-identité de deux objets, deux occurrences ou deux faits, qui traduit une erreur de jugement ou d'appréciation:

Ändern ist nicht Bessermachen

Fermer les yeux n'est pas toujours dormir. MPS230

L'insistance sur l'identité entre deux réalités apparemment sans commune mesure:

Nicht geschossen ist auch gefehlt

Ne rien dire, c'est consentir.

4. L'usage formel de conditions dont la réalisation est tenue pour invraisemblable:

Wenn man zu jeder Lüge pfeifen wollt', müsste man alleweil ein gespitztes Maul machen.

Quand les chats siffleront, à beaucoup de choses nous croirons. 
5. La formule de prévoyance Wer dies (nicht) ist/hat/macht, muss/soll das (nicht) machen / Qui fait /n'est / n'a (pas) ceci, (ne) doit (pas) faire cela:

Wer barfuss geht, soll keine Dornen säen (62)

Wer von Wachs ist, muss nicht zum Feuer kommen (648)

Qui a peur des étincelles ne devient pas forgeron. MPS233

Qui a une tête en beurre ne doit pas s'approcher du four. MPS238

6. L'usage des formules de comparaison: besser-als ou lieber als/mieux (vaut)-que Ici les comparés sont souvent des adjectifs, des substantifs, des infinitifs, des propositions:

Besser arm mit Ehren als reich mit Schande (117)

Besser, es fresse mich ein Wolf als ein Schaf (weil ein Wolf es schneller macht).

Besser den Arm brechen als den Hals (48)

Mieux vaut perdre la laine que le mouton. MPS200

Mieux vaut endiguer un ruisseau qu'une rivière. MPS243

Mieux vaut être piqué par une ortie que par une rose. Maloux 22

7. Le moule sémi-comparatif halb, halber, der halbe

Frisch gewagt ist halb gewonnen.

Ordnung ist das halbe Leben (243)

Chose bien commencée est à demi achevée. MPS71

À bon départ, cuvre à moitié faite. MPS202

8. Le moule qui exhorte à ne pas se fier aux apparences: L'image qui est donnée n'est pas la réalité.

Es ist nicht alles Gold, was glänzt (30)

Nicht alles, was süß ist, ist Honig (31)

Tout ce qui est blanc n'est point farine. MPS241

Tous ne sont pas des saints qui vont à l'église. MPS199

9. Es gibt viele..., aber wenig / nombreux, mais peu. Cette formule juxtapose la quantité initiale à la quantité réelle des agissants afin d'être plus parlante:

Es gibt viele, die mit dem Kreuz gehen, aber wenig Kreuzträger.

Beaucoup d'appelés mais peu d'élus.

10. L’opposition de deux actions en évoquant le contraire pour souligner ce qui est recommandé ou inversement: Es ist bös, übel / es ist gut, leicht:

Ohne Mehl und Wasser ist übel backen.

Im trüben Wasser ist gut fischen.

On ne peut pas manger et siffler.

Il est bon de pêcher en eau trouble.

11. L'usage du moule solch... solch / tel... tel pour marquer la correspondance entre deux entités:

Auf solchem Markte solcher Zoll.

Auf einen solchen Topf gehört ein solcher Deckel.

Tel arbre, tel fruit.

Telle marmite, tel couvercle.

12. Was hilft / was nutzt... ohne? / À quoi sert-il de faire ceci, si on ne / n'a pas fait cela?

Was hilft's, wenn man mit dem Kopf gegen die Wand rennt (326)

Il ne sert à rien de montrer les dents lorsqu'on est édenté. MPS37

13. L'usage de l'impératif au lieu du futur:

Singe, so lernst du singen!

Stiehl einmal und bleib dein Lebtag ein Dieb!

Si tu veux être un bon écrivain, écris! 


\section{Bist du, so / si tu es..., alors}

Bist du ein Narr, so lass dir eine Kappe machen.

Qui est un âne et veut être cerf se connaît au saut du fossé. MPS26

Cet inventaire n'est pas exhaustif mais il présente l'avantage de donner une vue générale des principaux modes d'élaboration des proverbes. Ceux-ci ayant été avant tout des phénomènes oraux. L'oralité est d'ailleurs l'un des moyens de communication par excellence dans les sociétés dites traditionnelles. Et si les proverbes sont permanents, et souvent stables dans leur morphologie, c'est bien en raison de facteurs formels comme le rythme. La structure rythmique marquée des proverbes les préserve donc de la précarité dont sont victimes les énoncés quotidiens dans l'oralité. Par structure rythmique, il faut entendre ici, l'ensemble des procédés prosodiques présents dans la majorité des proverbes et qui permettent de les distinguer des autres formes de discours. Le rythme se manifeste par le retour périodique d'éléments marqués: l'intonation, la symétrie dont la fonction est à la fois «mnémonique et incantatoire» (Rodegem 1974:123) et également par l'inférence (Mejri 2004). Le rythme, ainsi considéré, peut s'exprimer à travers plusieurs cas de figure comme les rimes (1), la symétrie des hémistiches (2), l'accentuation et la hauteur mélodique, puis le parallélisme phonique de deux ou plusieurs pieds dans un proverbe (3):

(1)

Wer auf Sand baut, hat umsonst gebaut. (485)

Beaucoup de bruits, peu de fruits. (Maloux 73)

Bhlèwô ne gwlè, ò gwè gwlè.

(Le bélier n'a pas longue vie, ses cornes ont longue vie)

Le bélier meurt mais ses cornes lui survivent.

(2)

Der Bock geht ein, aber seine Hörner überdauern ihn.

Reden ist Silber, Schweigen ist Gold (225)

Eil pour cil, dent pour dent. MPS36

ò gbisi gbê, ō gbisi lu.

Qui tire le panier, tire aussi la corde.

(3)

Wer den Korb heran zieht, zieht unbedingt auch am Seil.

Andere Länder, andere Sitten.

Loin des yeux, loin du cour. (Maloux 1)

Tèbhè ne nimi, wā piô bhló me.

(Le serpent ne mord pas, on ne le porte pourtant pas au cou.)

Même si l'on t'a dit que le serpent ne mord pas, ce n'est pas une raison pour le porter autour du cou.

Binde dir die Schlange nicht um den Hals, auch wenn man sagt, sie beiße nicht.

En effet, lorsque le proverbe est bien rythmé comme c'est le cas avec les unités suivantes,

Der eine sagt weiß, der andere schwarz (124)

Einem geschenkten Gaul, schaut man nicht ins Maul (191)

La mauvaise plaie se guérit, la mauvaise réputation tue. MPS210

Lampe placée trop haut sera soufflée par le vent, lampe placée trop bas soufflée par les enfants. MPS232 
ò kpeti sōkwlā sô, mesi fù ò đi.

Qui pourchasse deux mangoustes à la fois n'accompagnera pas de viande son plat de bananes.

Wer auf zwei Mangusten Jagd macht, der isst die Bananen ohne Fleisch.

la mémorisation par les locuteurs en est rendue facile. Il se crée implicitement un automatisme mental, auditif et verbal, car les locuteurs peuvent se rappeler ces formes et les reproduire plus aisément dans leurs conversations. Qui plus est, des proverbes marqués de la sorte ont plus de prégnance et davantage de possibilités de produire l'effet recherché par l'émetteur. D'autre part, c'est ce marquage rythmique qui, entre autres critères, permet au récepteur de déceler l'intention communicative de son interlocuteur. Et en réalité, avec de tels proverbes, l'émetteur peut même se permettre de ne pas énoncer entièrement la formule: Einem geschenkten Gaul..., un homme averti...sa structure permettra à celui qui l'entend de compléter implicitement le proverbe, les parties évoquées inférant de fait les membres tronqués.

Ainsi, traduire le proverbe, c'est chercher à répercuter le contenu conceptuel dénoté, mettre fidèlement en évidence l'image sur laquelle repose le concept en conservant l'amplitude de sa fonction symbolique. Ce sont ces principaux paramètres qui ont officié à l'élaboration de notre corpus de proverbes bété qui visait à dégager pour des champs conceptuels identiques définis à partir de l'allemand, un concordancier de proverbes. Sur cette base, nous avons établi un corpus expérimental parallèle reposant sur 83 champs conceptuels avec respectivement 604 proverbes allemands, 1120 proverbes en français et 309 proverbes en bété (Zouogbo 2005). Le jeu des traductions a fournit pour ces trois langues plusieurs niveaux de concordances dont nous exposons, ici, quelques-unes parmi les plus expressives.

\section{Mise en contraste des concordances totales}

La concordance parémiologique est dite totale lorsque la même idée, le même concept est illustré dans les deux langues avec les mêmes images, en l'occurrence Eisen/ fer et selon une structure équivalente. Il existe ainsi une concordance totale entre le français et le bété pour les proverbes suivants.

Les doigts d'une main ne s'entresemblent pas. MPS37

Sòwleyè ne yulèleku.

Les doigts de la main n'ont pas la même taille. Ils ne se ressemblent pas.

Die Finger einer Hand sehen nicht alle gleich aus.

Nous présenterons, dans ce qui suit, les proverbes en fonction de leur productivité autour de quelques concepts spécifiques retenus, afin de voir comment les mêmes concepts sont exprimés et au moyen de quelles images. On restera ainsi attentif à la transcription faite des proverbes bété et leur traduction en allemand puis en français. Sera marquée entre parenthèses, juste en dessous du proverbe bété, la transcription littérale d'origine. La seconde traduction est notre lecture personnelle et une traduction adaptée autant que possible aux canons morphologiques conventionnels. On constatera ainsi les affinités de principe que nous avions énoncées au niveau de la forme et du contenu. 


\subsection{Allemand/Bété}

\section{SOLIDARITÉ, UNION / SOLIDARITÄT, EINTRACHT}

Eine Hand wäscht die andere (38)

Dilikwlèsô bhudualô kòpèsô.

(C'est la main avec laquelle on mange/ qui lave / la main gauche.)

La main droite lave la main gauche.

Die rechte Hand wäscht die linke.

2. ADAPTATION / DIE ANPASSUNGSFÄHIGKEIT

Wo die Ziege angebunden ist, da muss sie grasen (37)

Wli, / de wā gbètole / lòbhô y'ô li.

(Le cabri, /là où il est attaché /c'est là qu'il broute.)

Le cabri broute là où il est attaché.

Wo es angebunden ist, da grast das Zicklein.

3. INTÉRÊTS / DIE EIGENEN INTERESSEN VERTRETEN

Es sägt niemand gern den Ast ab, auf dem er sitzt (50)

E kà suklê kugli ne'cè kudi.

(Tu ne peux être assis sur une branche et puis la couper.)

Tu ne peux couper une branche sur laquelle tu es assis.

Den Ast, auf dem du sitzt, kannst du nicht absägen.

\section{VÉRITÉ et MENSONGES / LÜGEN und WAHRHEIT}

Kindermund tut die Wahrheit kund (309)

Yuā ne la yò nu kè.

(Les enfants n'ont pas de mensonges dans la bouche.)

Les enfants ne mentent jamais.

Kinder lügen nie.

5. NE PAS GÉNÉRALISER / (MAN SOLLTE) NICHT VERALLGEMEINERN

Eine Fichte macht keinen Wald (158)

Eine Tanne macht keinen Wald (579)

E ke su bhló ga, e nā cèbhè gā.

(Quand tu évoques un seul arbre, ce n'est pas de toute la forêt que tu parles.)

Un seul arbre ne fait pas la forêt.

Meinst du einen Baum, so meinst du aber nicht den ganzen Wald.

\subsection{Français /Bété}

\section{VANITÉ / EITELKEIT}

Les oreilles ne poussent pas plus haut qu'on a le front. MPS253

Jukuli ne kpōmā ne è ke wuli kùzi.

(L'oreille ne pousse pas jusqu'à dépasser la tête.)

L'oreille a beau grandir, elle ne dépassera jamais la tête.

Wie groß das Ohr auch werden mag, es wird nie über den Kopf hinaus-

wachsen.

\section{ADAPTATION / DIE ANPASSUNGSFÄHIGKEIT}

Où la chèvre est liée, il faut qu'elle broute. MPS30

Wli, de wà gbètole lòbhô y'ô li.

Le cabri broute là où il est attaché.

Wo es angebunden ist, da grast das Zicklein.

\section{INÉGALITÉS NATURELLES / NATÜRLICHE UNGLEICHHEITEN}

Les doigts d'une main ne s'entresemblent pas. MPS37

Sòwleyè ne yulèleku.

(Les doigts de la main n'ont pas la même taille.)

Les doigts ne se ressemblent pas.

Die Finger einer Hand sehen nicht alle gleich aus. 


\section{POSER DES ACTES INUTILES / UNNÖTIG HANDELN}

Au poisson à nager ne montre. MPS18

Wā ne ciè zibhì yudruè.

On n'apprend pas à nager à un poisson.

Einem Fisch bringt man das Schwimmen nicht bei.

5. VÉRITÉ et MENSONGES / LÜGEN und WAHRHEIT

La vérité sort de la bouche des enfants. MPS36

Yuā ne la yò nu kè.

Les enfants n'ont jamais de mensonges (dans la bouche).

Les enfants ne mentent jamais.

Kinder lügen nie.

\section{DESTIN / SCHICKSAL}

Dieu a tout dans une main. MPS247

Làgō y'è kolô kò.

C'est dans les mains de Dieu que les choses sont.

Tout est dans les mains de Dieu.

Gott hat alles in seinen Händen. (Alles liegt in Gottes Händen.)

7. BESOIN, NÉCESSITÉ / BEDÜRFNIS, NOT

Ventre affamé n'a point d'oreilles. MPS38

Il est difficile de discuter avec le ventre, car il n'a pas d'oreilles. (Maloux 179)

yonhüyimle ne yukwli kè.

(Le ventre qui connaît la faim n'a pas d'oreilles.)

Ventre affamé n'a point d'oreilles.

8. CHÂTIMENT / STRAFE

Ein hungriger Bauch hat keine Ohren.

Qui crache vers le ciel est atteint lui-même. MPS237

ò wale nògô me dô, oyi ye dô srū kló ku.

(Celui qui crache dans le ciel, c'est sur son visage que verse la salive.)

Qui crache en direction du ciel, reçoit sa propre salive en plein visage.

Wer zum Himmel spuckt, der bekommt die eigene Spucke ins Gesicht.

\section{Conclusion}

Les recherches en parémiologie ont connu un essor prodigieux ces dernières décennies. Les travaux comparés à visée interculturelle y ont largement contribué et continuent de constituer un domaine porteur d'intérêt. Dans cette perspective, les analyses qui portent sur la mise en contraste de plusieurs langues sur la base des proverbes prennent pour point de départ la constitution de corpora parallèles. Pour les langues élaborées, les choix se portent d'emblée sur les sources existantes. Pour les langues encore au stade de codification, la liberté de manœuvre est restreinte. Le fait est qu'il n'existe pas encore de sources parémiographiques. Il incombe, par conséquent, aux chercheurs de répertorier les proverbes puis de les traduire dans une langue étalon. Il ne s'agira plus alors de transposer systématiquement les énoncés d'une langue à l'autre mais de contribuer à une lecture correcte des proverbes en répercutant de manière idoine le contenu conceptuel de ces formules sans trahir la fonction symbolique des images présentes. Car traduire le contenu conceptuel, c'est rester fidèle à l'identité sémantique du proverbe. Il est intéressant en outre d'essayer de se conformer aux principaux modèles morphologiques qui consacrent les différences notables, parmi les syntagmes phrastiques, entre ce qui peut être considéré comme un proverbe ou ce qui ne l'est pas. 
Au delà des enjeux interculturels, la problématique de la traduction du proverbe demeure un phénomène bien connu des traducteurs professionnels. Il s'agit de séquences figées qui se retrouvent de plus en plus dans les textes. Quand l'on a déjà rencontré l'une ou l'autre forme et que l'on en sait le concordant dans la langue d'arrivée, la tâche de la traduction est mécanique. L’enjeu est tout autre lorsque la formule en présence est méconnue. La traduction devra alors être axée sur la transmission du contenu conceptuel de la séquence. Ici, la méconnaissance de la valeur et de la fonction symbolique spécifique des images utilisées peut être un grand facteur d'incertitude.

\section{RÉFÉRENCES}

Anscombre, J.- C. (2003): «La parole proverbiale et structure métrique», Langage 139, pp. 6 38.

Beyer, A. und H. Beyer (1990): Sprichwörterlexikon, C-Beck, München.

Burger, H. (2003): Phraseologie, Erich Schmidt, Berlin.

BURIDANT, C (1976): «Nature et fonction des proverbes dans les jeux parties», Revue des sciences humaines 163.

Buridant, C (1984): Les proverbes et la prédication au Moyen Age, Presses universitaires, Lille GréCIANO, G. (1984): «Pour un apprentissage des unités phraséologiques», Nouveaux Cahiers d'Allemand 2, p. 95-113.

Maloux, M. (1997): Dictionnaires des proverbes, sentences et maximes, Larousse, Paris.

Rodegem, F. (1984): «La parole proverbiale», in SuArd, F. et C. Buridant (dir.), Richesse du proverbe, Presses universitaires, Lille.

SEILER, F. (1922): Sprichwörterkunde, München, Beck.

Zouogbo, J- P. (2005): Concepts et images parémiologiques. Une étude de linguistique comparée allemand/français/bété, Thèse de doctorat, Strasbourg et Bâle. 